\title{
Original
}

\section{An Experimental Carious Detector to Stain the Carious Infected Dentin}

\author{
Misa OIKAWA, Mizuho KUSUNOKI, Kazuo ITOH \\ and Hisashi HISAMITSU \\ Department of Clinical Cariology and Endodontology, \\ Showa University School of Dentistry
}

\begin{abstract}
Objectives: The purpose of this study was to develop an experimental carious detector by which only carious-infected dentin is stained and to investigate bacterial invasion after removal of dentin based on staining using the experimental carious detector. Methodology: Micro Vickers Hardness (MVH) of the extracted human teeth was measured on the longitudinal section surface from the pulp chamber to the carious region using a micro hardness tester. The carious dentin was removed based on tactile sensitivity, and based on staining using conventional and experimental carious detectors. The MVH of the remaining dentin cavity wall was determined by observing the indentation beneath the cavity wall. The microstructure of the remaining dentin was observed using a SEM (S-4700, Hitachi, JAPAN) and the possibility of bacteria survival was examined using a Gram stained specimen. Results: The

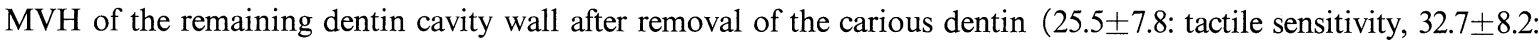
experimental carious detector, $44.7 \pm 6.6$ : conventional carious detector) was statistically significantly different by ANOVA with Fisher's PLSD $(p<0.001)$. The dentin tubules of the cavity wall were completely closed with rod-like substances with a cubed structure after the carious dentin was removed using the experimental carious detector. In addition, no bacteria was observed in any of the specimens. Conclusion: It was possible to conclude that the carious-infected dentin was completely removed and sclerotic dentin was preserved under the guidance of the experimental carious detector stain composed of poly-propylene glycol and acid red.
\end{abstract}

Key words: carious dentin, carious detector, poly-propylene glycol.

It was reported that two layers were identified in etched canine dentin. ${ }^{1)}$ To distinguish these two layers, Terashima et al. invented a carious detector composed of basic fuchsine diluted in propylene glycol in 1972.2) Fusayama suggested that the carious detector stained only the outer layer of carious dentin that could not re-calcify. In addition, they claimed that the inner layer of the carious dentin should be preserved because it re-calcified using proper dental treatment. ${ }^{3)}$ This ability for re-calcification was explained through the biochemical experiments of Kuboki et al. ${ }^{4)}$ They defined that the cross linking of the dentin collagen was destroyed and irreversibly degraded in the outer layer of the carious dentin whereas it was possible for the inner layer to recover. The red dye in this solution was changed from basic fuchsine to acid red because the former was possibly carcinogenic.5) However, the clinical application time of this carious detector was not exactly specified and the stained substance in the dentin has not been clarified. It is clear that the carious detector stains sound dentin if applied to the cavity over a long time because it is useful as a tracer in the marginal leakage test. Furthermore, although clinical treatment for pink-stained dentin is still being discussed, no consistent conclusion has been reported. ${ }^{6 \sim 8)}$ The sclerotic dentin is considered to be preserved because the dentin tubules are filled with the Whitlokite to prevent bacterial invasion. We demonstrated that the bonding efficacy of the dentin bonding system was superior in sclerotic dentin compared to that in sound dentin because the adhesive monomer concentration was maintained at a high level at the adhesive interface. ${ }^{9 \sim 11)}$ In addition, we reported that sclerotic

(Received September 6, 2007;Accepted for publication January 21, 2008) 
Table 1 The conventional Carious Detector and the variety of the experimental detector.

Carious Detector (Kuraray)

Experimental carious detector (134)

Experimental carious detector (192)

Experimental carious detector (300) (propylene glycol)

(di-propylene glycol)

(tri-propylene glycol)

(poly-propylene glycol)
( ): Molecular weight

dentin was removed completely when pink-stained dentin was removed ${ }^{12)}$. However, it is impossible to leave the pink-stained dentin because the stained dentin detracted from the esthetic characteristics of the resin composite restoration.

The purpose of this study was to develop an experimental caries detector composed of acid red and poly-propylene glycol where the sclerotic dentin was not stained and to investigate bacterial invasion into the dentin after removing the dentin stained by the experimental carious detector.

\section{Materials and Methods}

The composition of the conventional carious detector and the experimental detector tested are listed in Table 1. The experimental carious detectors were prepared by diluting acid red in di-propylene glycol, tri-propylene glycol or poly-propylene glycol solution at a concentration of $1 \mathrm{wt} \%$.

\section{SEM observation}

SEM observation of the cavity wall after removing dentin stained by the carious detector.

The carious dentin in the extracted human molar teeth was removed using a round-shaped steel bur mounted on a low-speed cutting machine under a water coolant. In the control group, the carious dentin was removed based on tactile sensitivity by the five-year clinical experienced dentist. In the experimental groups, one of either the conventional or experimental carious detectors was applied into the cavity for a few seconds followed by rinsing and drying. The stained dentin was then completely removed using a round-shaped steel bur as described above. After removing the stained dentin, the carious detector was applied again to the cavity and the stained dentin was completely removed. This method of detector application of the carious detector and removal of the stained dentin was repeated until the cavity wall was no longer stained. The cavity wall was then treated with $0.5 \mathrm{~mol} / \mathrm{L}$ EDTA solution for
$60 \mathrm{~s}$ to remove the smear layer. Then the teeth were dehydrated in gradual alcohol solutions and critical point dried. The microstructure of the dentin was observed using SEM after vacuum sputtering the specimen with platinum and palladium.

\section{Micro Vickers Hardness measurement}

The forty five extracted human teeth with carious dentin were sectioned though the center of the carious along the longitudinal axis. The Micro Vickers Hardness of the dentin was measured from the pulp chamber wall to the carious cavity every $200 \mu \mathrm{m}$ under the condition of $50 \mathrm{~g}$ loading for $20 \mathrm{~s}$. The carious dentin was then stained using Caries Detector or the experimental detector containing polypropylene glycol and the stained dentin was removed completely using the round bur as described above. After removing the stained dentin completely, the hardness of the cavity wall was determined by observing the indentation beneath the cavity wall. For the control, the carious dentin was removed based on the tactile sensitivity. In total, fifteen specimens were measured for each detector.

\section{Investigation of the bacterial invasion}

The carious dentin of the extracted human teeth was removed using the same method for the SEM observation specimens. The teeth were then decalcified in EDTA solution for three weeks and in formic acid for two weeks. The decalcified teeth were embedded in paraffin and sliced to a thickness of approximately $5 \mu \mathrm{m}$. After eliminating the paraffin, the specimens were stained using crystal violet, rugols solution followed by safranine. Bacterial invasion into the cavity wall was observed under light microscope.

\section{Results}

SEM observation of the cavity wall after removing the stained dentin

When the carious dentin was removed based on tactile sensitivity, the dentin tubules in the cavity wall 
were filled with crystal-like substances (Figure 1-a) whereas these were completely open where dentin was removed based on conventional carious detector staining (Figure 1-b). When the carious dentin was removed based on experimental detector staining, about half of the dentin tubules were filled with debris (Figure 2-a, b) and almost of the dentin tubules were filled with crystals when the polypropylene glycol (molecular weight of 300) was used

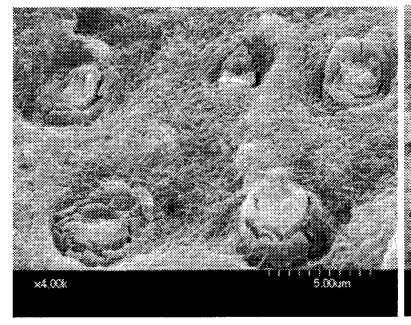

a

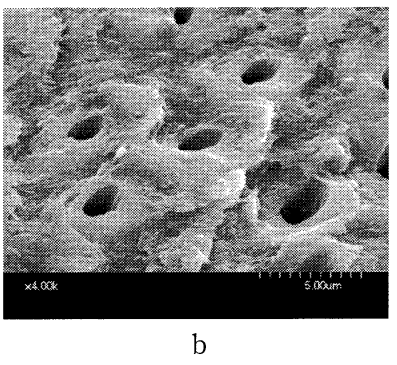

b
Fig. 1 SEM observation of the cavity wall. a: Carious dentin of the specimen was removed based on tactile sensitivity. The dentin tubules were filled with a crystal like substance. b: SEM observation of the cavity wall. Carious dentin of the specimen was removed based on conventional carious detector staining. The dentin tubules were completely open.

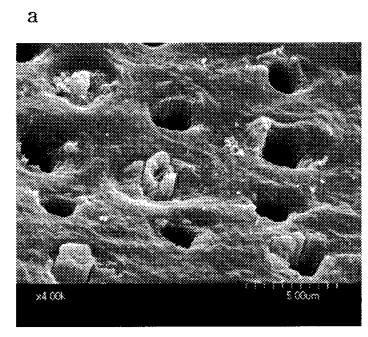

b
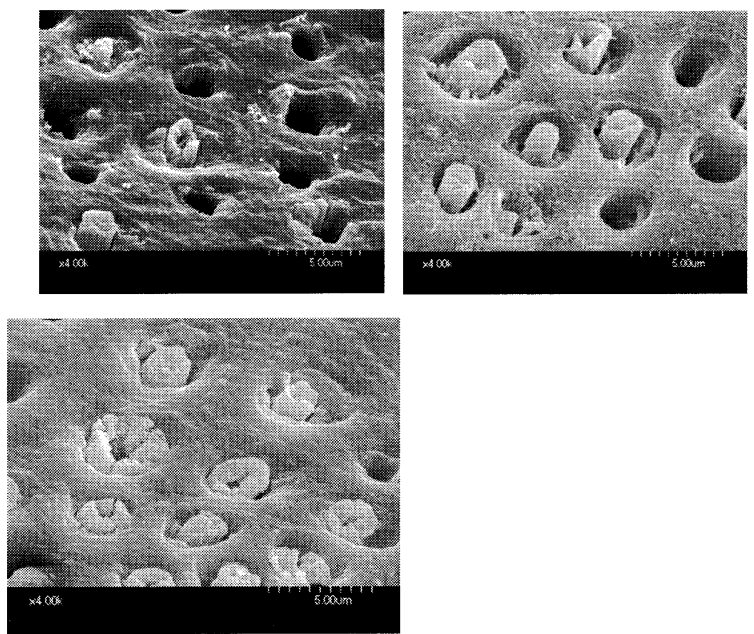

Fig. 2 SEM observation of the cavity wall. a, b: Carious dentin of the specimen was removed based on experimental detector staining. (dipropylene glycol: Fig. 2-a; tri-propylene glycol: Fig. 2-b) About half of the dentin tubules were filled with a crystal like substance. c: Carious dentin of the specimen was removed based on the experimental detector containing polypropylene glycol. Almost all of the dentin tubules were filled with a crystal like substance.
(Figure 2-c).

MVH of the cavity wall after removing the dentin stained by the carious detector

When the dentin stained by the conventional carious detector was completely removed, the MVH on the cavity wall was higher than that of the specimen in which the dentin was removed based on the experimental detector or tactile sensitivity. The MVH of the three groups were statistically significantly different (Figure 3). As shown in Figure 4, the MVH of the conventional detector specimens was as high as that of the sound dentin (Figure 4).

\section{Observation of the Gram stained specimens}

Violet-stained Streptococci were observed in the carious lesions of the extracted human teeth (Figure 5). In this study, Streptococci etc. was not observed in

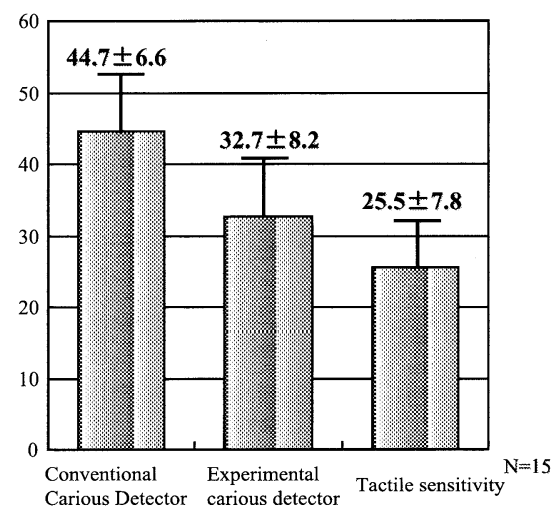

(The One-way ANOVA Fisher's PLSD $(\mathrm{p}<0.001)$ )

Fig. 3 Micro Vickers Hardness (MVH) of cavity wall. The MVH on the cavity wall was highest when the dentin stained by the conventional carious detector was completely removed. The MVH of the three groups tested was statistically significantly different by One-Way ANOVA with Fisher's PLSD ( $p<0.001)$.

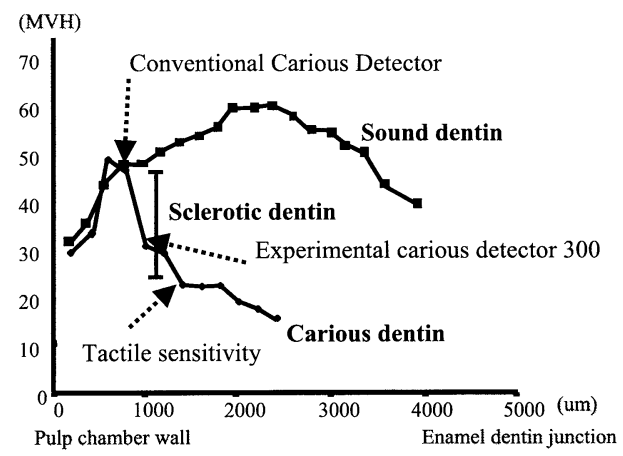

Fig. 4 Micro Vickers Hardness (MVH) from the pulp chamber wall to the enamel dentin junction. 


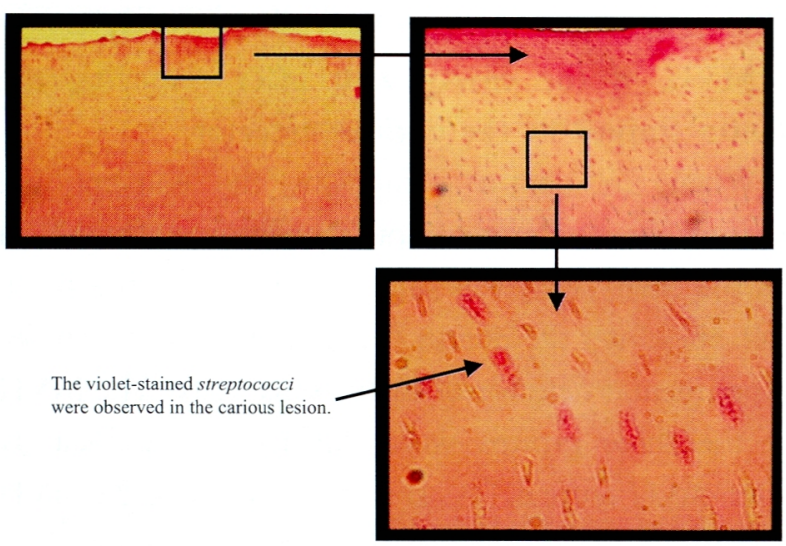

Fig. 5 Observation of the Gram stained specimen that was the extracted human carious tooth. Streptococci were observed in dentin tubules. In inter- and peri- tubular dentin they were not observed.

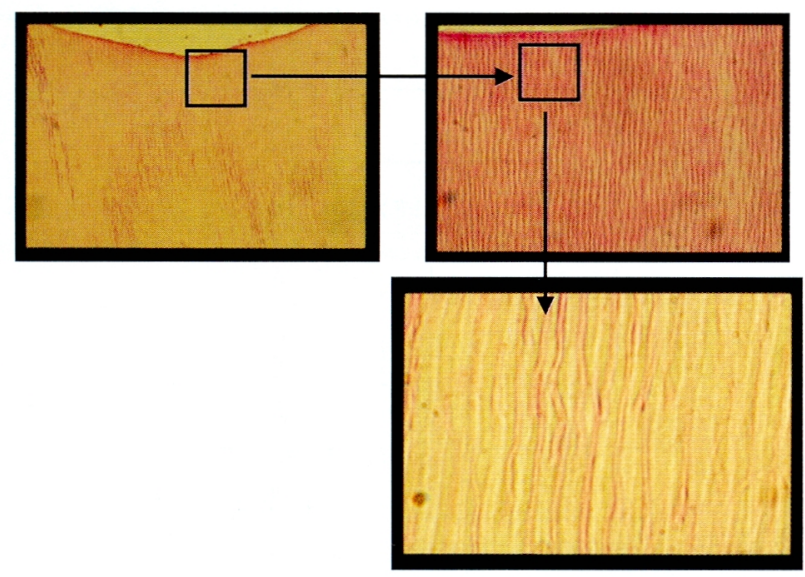

Fig. 6 Observation of the Gram stained specimen that was carious dentin removed based on tactile sensitivity. Streptococci were not observed.

any of the specimens where the carious dentin was removed based on tactile sensitivity and under the guidance of the staining of the carious detector regardless of the composition of the detector employed (Figures 6, 7, 8).

\section{Discussion}

To remove the carious-infected dentin, it is widely recognized that the softened dentin should be removed in a clinic because the foremost softened dentin is deeper in the cavity than the foremost discoloration and bacterial invasion. ${ }^{13)}$ The carious detector invented by Fusayama et al. was reported to stain only the outer layer of the carious dentin that can not re-calcify. ${ }^{3)}$ However, as demonstrated in this

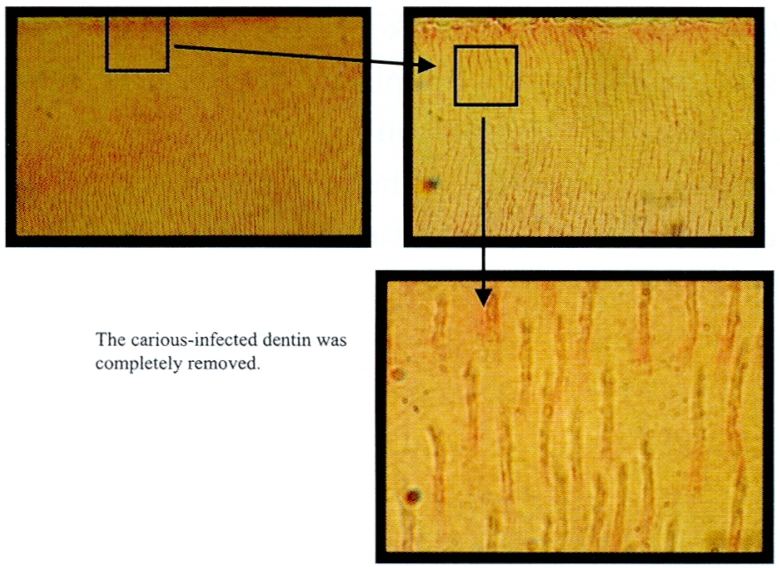

Fig. 7 Observation of the Gram stained specimen that was carious dentin removed based on experimental detector staining. Streptococci were not observed.

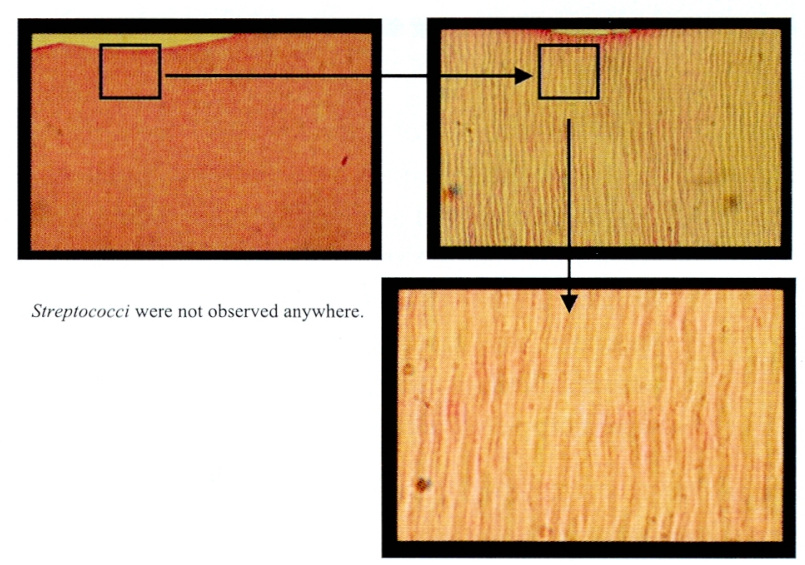

Fig. 8 Observation of the Gram stained specimen that was made of carious dentin removed based on conventional carious detector staining. Streptococci were not observed.

study, the propylene glycol penetrated through the sclerotic dentin close to the sound dentin. The experimental carious detector containing poly-propylene glycol did not stain the sclerotic dentin, probably because of the higher molecular weight compared to that of propylene glycol. The function of the red dyestuff in the carious detector is limited to demonstrating the penetration of the detector for the naked eye in a clinic.

As demonstrated in this study, bacterial invasion was not detected in any of the specimens in which the carious dentin was removed based on conventional or experimental carious detector staining. Therefore, it is possible to conclude that the experimental detector containing poly-propylene glycol was effective in removing the carious-infected dentin 
completely and preserving the sclerotic dentin.

The bonding between the dentin and dentin adhesive has been explained by hybrid formation of the adhesive monomer into the superficial substrate dentin. ${ }^{14)}$ The dentin adhesive was considered to penetrate the dentin collagen in its expanded state due to the moist condition of the dentin priming, though most adhesive monomers never had a chemical structure to bond to the organic component. ${ }^{15,16)}$ In addition, the dentin adhesive exhibited higher bonding efficacy to the enamel compared to that of the dentin despite the extremely low collagen content of the enamel. Most of the explanations stating that the bonding agent bonded to the dentin collagen were based on the bond strength measurement of the dentin adhesive to the flat dentin surface. In these organic penetration bonding reports, it was impossible to detect the interaction between the polymerization contraction stress and the efficacy of the dentin adhesive.

We evaluated the efficacy of the dentin adhesives by observing the contraction gap in the cylindrical dentin cavity as suggested by Asmussen. ${ }^{17)}$ It was convincingly concluded that the formation of a gap was prevented by the inorganic component of the dentin and the adhesive monomer in the dentin bonding agent because a gap was observed when the dentin cavity wall was etched using a dentin conditioner when the adhesive monomer was omitted from the dentin bonding agent. ${ }^{18)}$ Furthermore, the dentin primer is essential to obtaining the marginal integrity of the resin composite. ${ }^{19,20)}$ In our previous reports, formation of a contraction gap of the resin composite in a cylindrical dentin cavity was prevented in half of the cavities prepared in the sclerotic dentin even when the cavity wall was not primed. The gap was not prevented in any of the specimens of the sound dentin cavity without priming. In addition, complete marginal adaptation was obtained when the cavity wall was primed by the glyceryl mono-methacrylate solution in both the sclerotic and sound dentin cavities. Therefore it was possible to speculate that the sclerotic dentin exhibited the desirable effect at the substrate for the dentin bonding because the monomer infiltration was restrained and a high monomer content at the adhesive surface was maintained in the sclerotic dentin. ${ }^{15)}$ Such monomer immersion into the dentin was further prevented by the dentin primer. In addition, it was possible to explain the priming effect through the disturbing of the adhesive monomer diffusion and the fluid flow through the dentin structure to the adhesive interface. Thus sclerotic dentin should be preserved because it is not infected and has an advantage as the substrate for dentin bonding compared to sound dentin.

As discussed above, it is possible to conclude that the carious-infected dentin was completely removed and sclerotic dentin was preserved under the guidance of the experimental carious detector stain composed of poly-propylene glycol and acid red.

Acknowledgments This investigation was supported by "High- Tech Research Center" Project for Private Universities: matching fund subsidy from MEXT (Ministry of Education, Culture, Sports, Science and Technology), 2005-2008.

\section{References}

1) Katou S : Recalcification of artificially decalcified dentine in living dog teeth. J Stomatol Soc Jpn, 35: 613-625, 1968

2) Terashima $S:$ Differentiation of the two layers of carious dentin by staining. J Stomatol Soc Jpn, 37: 279-286, 1970

3) Fusayama $T$, Terashima $S$ : Differentiation of two layers of carious dentin by staining. Bul Tokyo Med Dent Univ, 19: 83-92, 1972

4) Kuboki $Y$, Ohgushi $K$, Fusayama $T$ : Collagen biochemistry of the two layers of carious dentin. J Dent Res, 56: 1233-1237, 1979

5) Fusayama T, Takatsu T, Kazuo I, Yamauchi J, Shibatani K : New composition of carious detector. Japan. J Conserv Dent, 22: 261-264, 1979

6) Sano H : Relationship between caries detector staining and structural characteristics of carious dentin. $\mathbf{J}$ Stomatol Soc Jpn, 54: 241-270, 1978

7) Kidd EAM, Joyston-Bechal S, Beighton D:The use of a carious detector dye during cavity preparation: a microbiological assessment. Brit Dent J, 174: 245-248, 1993

8) Hukusima $M$ : Adhesive resin penetration into caries dentin. J Stomatol Soc Jpn, 48: 362-385, 1981

9) Tani C, Itoh K, Hisamitsu H, Wakumoto S : Efficacy of dentin bonding to cervical defects. Dent Mater J, 20: 359-368, 2001

10) Kusunoki M, Itoh $K$, Hisamitsu $H$, Wakumoto $S$ : The efficacy of dentine adhesive to sclerotic dentin. J Dent, 30: 91-97, 2002

11) Wu J, Itoh $\mathrm{K}$, Yamashita $\mathrm{T}$, Hisamitsu $\mathrm{H}$, Wakumoto $\mathrm{S}:$ The efficacy of the dentin adhesive to the 
caries-affected dentin. J Showa Univ Dent Soc, 23: 166-174, 2003

12) Kusunoki M, Oikawa M, Itoh $K$, Hisamitsu $H$ : SEM observation of the dentin staining with carious detector. J Dent Res, 81: (Special Issue A) \#0593 A-98 (Abstract), 2002

13) Okuse $K$ : Relationship between hardness, discoloration and microbial invasion in carious dentin. $\mathrm{J}$ Stomatol Soc Jpn, 31: 187-200, 1964

14) Nakabayashi N, Kojima $K$, Masuhara $E$ : The promotion of adhesion by the infiltration of monomers into tooth substrates. J Biomed Mater Res, 16: 265-273, 1982

15) Sugizaki J : The effect of the various primers on the dentin adhesion of resin composites-SEM and TEM by observation of the resin impregnated layer and adhesion promoting effect of the primers. Japan J Conserv Dent, 34: 228-265, 1991
16) Gwinnett AJ, Kanca JA : Micromorphology of the bonded dentin interface and its relationship to bond strength. Am J Dent, 5: 73-77, 1992

17) Asmussen $\mathrm{E}$ : Composite restorative resins; Composition versus wall-to-wall polymerization contraction. Acta Odontol Scand, 33: 337-344, 1975

18) Chiba $M$, Itoh $K$, Wakumoto $S$ : Effect of dentin cleansers on the bonding efficiency of dentin adhesive. Dent Mater J, 8: 76-85, 1989

19) Manabe A, Itoh K, Tani C, Hisamitsu H, Wakumoto $\mathrm{S}$ : Effect of functional monomer in commercial dentin bonding agents use of an experimental dentin bonding system. Dent Mater J, 18: 116-123, 1999

20) Chigira $H$, Manabe $A$, Itho $K$, Wakumoto $S$ : Efficacy of glyceryl methacrylate as a dentin primer. Dent Mater J, 8: 194-199, 1989 